\title{
LIDERANÇA IDEAL E REAL DOS ENFERMEIROS DE UNIDADE DE TERAPIA INTENSIVA EM HOSPITAIS PRIVADOS E PÚBLICOS*
}

\author{
Alexandre Pazetto Balsanelli ${ }^{1}$, Isabel Cristina Kowal Olm Cunha ${ }^{2}$
}

\begin{abstract}
RESUMO: Objetivou-se analisar a diferença nos estilos de liderança ideal e real dos enfermeiros de unidades de terapia intensiva de hospitais privados e públicos. Estudo transversal realizado em quatro unidades de terapia intensiva (duas privadas e duas públicas) do município de São Paulo, SP, Brasil. A amostra foi constituída por 66 duplas (enfermeiros e técnicos em enfermagem) definida por sorteio. A coleta de dados ocorreu no período de janeiro a abril de 2013. Os enfermeiros responderam ao questionário Grid \& Liderança em Enfermagem - comportamento ideal. Os técnicos em enfermagem ao questionário Grid \& Liderança em Enfermagem - comportamento real, considerando o enfermeiro que o sorteou. Dentre os 66 enfermeiros, $65(98,5 \%)$ consideraram o estilo 9,9 como ideal e $42(63,6 \%)$ foram avaliados como 9,9 pelo técnico em enfermagem. Não houve diferença entre os estilos de liderança ideal e real dos enfermeiros das unidades de terapia intensiva de hospitais privados e públicos ( $\mathrm{p}=0,67)$.
\end{abstract}

DESCRITORES: Enfermagem; Liderança; Unidades de Terapia Intensiva.

\section{IDEAL AND REAL LEADERSHIP OF NURSES IN INTENSIVE CARE UNITS AT PRIVATE AND PUBLIC HOSPITALS}

\begin{abstract}
The aim of this study was to analyze differences in styles of ideal and real leadership of nurses in intensive care units at private and public hospitals. A cross-sectional study was carried out in four intensive care units (two private and two public) in the city of São Paulo, state of São Paulo, Brazil. The sample consisted of 66 pairs (nurses and nursing technicians) randomly chosen. Data were collected between January and April of 2013. The nurses answered the questionnaire Grid \& Leadership in Nursing - ideal behavior. The nursing technicians answered the questionnaire Grid \& Leadership in Nursing - real behavior, considering the nurse who chose them. Among the 66 nurses, $65(98.5 \%)$ considered the style 9.9 as ideal and $42(63.6 \%)$ were evaluated as 9.9 by the nursing technician. There were not differences between nurses' ideal and real styles of leadership in intensive care units at private and public hospitals $(\mathrm{p}=0.67)$.
\end{abstract}

DESCRIPTORS: Nursing; Leadership; Intensive Care Units.

\section{LIDERAZGO IDEAL Y REAL DE ENFERMEROS DE UNIDAD DE TERAPIA INTENSIVA EN HOSPITALES PRIVADOS Y PÚBLICOS}

\begin{abstract}
Se objetivó analizar diferencias en estilos de liderazgo ideal y real de enfermeros de unidades de terapia intensiva de hospitales privados y públicos. Estudio transversal, realizado en cuatro unidades de terapia intensiva (dos privadas, dos públicas) del municipio de São Paulo-SP, Brasil. Muestra constituida por 66 duplas (enfermero y técnico en enfermería), definidas por sorteo. Datos recolectados de enero a abril de 2013. Los enfermeros respondieron el cuestionario Grid \& Liderazgo en Enfermería - comportamiento ideal. Los técnicos de enfermería, el cuestionario Grid \& Liderazgo en Enfermería - comportamiento real, considerando al enfermero que lo sorteó. De los 66 enfermeros, 65 (98,5\%) consideraron el estilo como ideal con puntaje 9,9; 42 (63,6\%) fueron puntuados con 9,9 por el técnico en enfermería. No hubo diferencia entre los estilos de liderazgo ideal y real de enfermeros de unidades de terapia intensiva privadas y públicas $(\mathrm{p}=0,67)$.
\end{abstract}

DESCRIPTORS: Enfermería; Liderazgo; Unidades de Cuidados Intensivos.

\footnotetext{
* Artigo extraído da Tese de Doutorado intitulada: “A liderança do enfermeiro em unidade de terapia intensiva e sua relação com o ambiente de trabalho". Escola Paulista de Enfermagem - Universidade Federal de São Paulo, 2014.

${ }^{1}$ Enfermeiro. Doutor em Enfermagem. Docente da Escola Paulista de Enfermagem. Universidade Federal de São Paulo. São Paulo, SP, Brasil. ${ }^{2}$ Enfermeira. Livre Docente. Pró Reitora de Administração da Universidade Federal de São Paulo. São Paulo, SP, Brasil.
} 


\section{- INTRODUÇÃO}

A liderança é uma competência extremamente necessária e requerida do enfermeiro. As instituições de saúde procuram profissionais que estejam aptos a exercê-la para atingirem resultados eficazes. Seu aprendizado inicia-se na graduação e continua permanentemente. Ao gerenciar equipe, receber e dar feedback, tomar decisões, solucionar conflitos, dentre outros, o enfermeiro desenvolve-se como líder.

A Unidade de Terapia Intensiva (UTI), no contexto hospitalar, torna-se um campo favorável para isto. Ao cuidar do paciente em estado crítico de saúde, gerenciar recursos para que a assistência ocorra com qualidade, exercer a interdisciplinaridade e ter contato com a família, o enfermeiro necessita exercer a liderança.

Os estudos sobre liderança em enfermagem na UTI têm abordado os seguintes aspectos: associação da liderança com a carga de trabalho(1), perfil pessoal e profissional ${ }^{(2)}$ e ambiente de trabalho $^{(3)}$; implantação de novos modelos ${ }^{(4)}$; percepção dos técnicos e auxiliares de enfermagem sobre o papel de líder desempenhado pelo enfermeiro ${ }^{(5)}$; experiências ${ }^{(6)}$ e reflexões ${ }^{(7)}$ sobre liderança vividas pelos enfermeiros de UTI e a liderança como competência ${ }^{(8)}$.

No entanto, para que o enfermeiro exerça o seu papel de líder, a instituição de saúde deve possuir um modelo de gestão que favoreça o seu exercício ${ }^{(9-10)}$. Tendo em vista que as pesquisas que buscaram verificar esta correlação ainda são poucas ${ }^{(3)}$ surge a questão deste estudo: "Existe diferença entre os estilos de liderança dos enfermeiros das UTI de hospitais privados e públicos?"

A resposta desta indagação permitirá entender como os enfermeiros de UTI praticam sua liderança no dia a dia e se esta sofre influência da organização. Desta forma, os gestores poderão traçar planos de ação para o aprimoramento desta competência considerando os modelos de gestão que regem os serviços hospitalares. Além disto, contribuir com a lacuna da literatura sobre esta temática tão importante no cenário da Enfermagem e também da unidade de terapia intensiva.

Os estilos de liderança dos enfermeiros têm sido analisados com o uso da Teoria do Grid Gerencial ${ }^{(11)}$. Esta não considera apenas uma tarefa particular, mas o contexto geral, ou seja, como o enfermeiro idealiza sua liderança e qual a visão dos liderados sobre como tem sido este exercício ${ }^{(11)}$. Sua adaptação para a Enfermagem, na qual o eixo horizontal indica o interesse pelos serviços hospitalares e o vertical a preocupação com os colaboradores, está demostrada na Figura $1^{(12)}$.

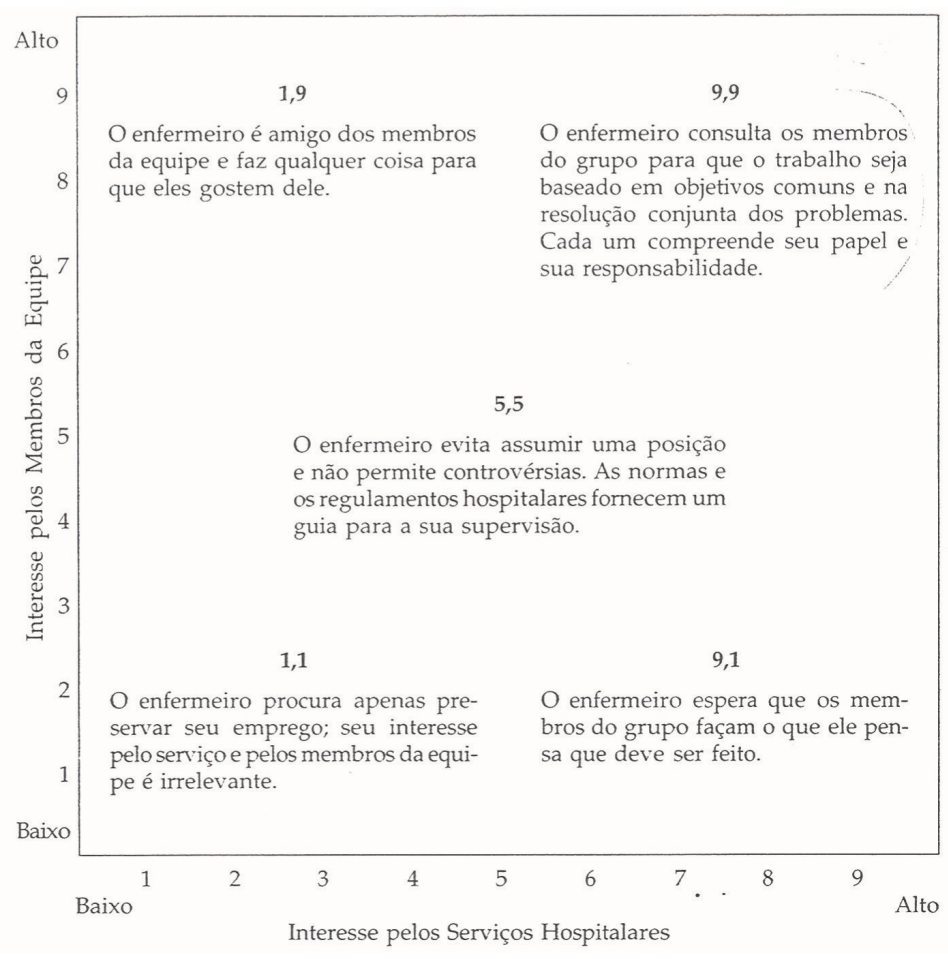

Figura 1 - Grid do Enfermeiro Administrador ${ }^{(12)}$. São Paulo - SP, Brasil, 2013 
Ante o exposto, o objetivo deste estudo foi analisar se existe diferença nos estilos de liderança ideal e real dos enfermeiros de unidades de terapia intensiva de hospitais privados e públicos.

\section{MÉTODO}

Estudo transversal realizado em quatro UTIs da zona sul do município de São Paulo, SP, Brasil. Resumidamente as características destas UTI são: pertencem a hospitais de nível terciário, são gerais com atendimento de pacientes adultos com afecções clínicas e cirúrgicas. Possuem respectivamente 42, 26, 32 e 30 leitos. Duas unidades pertencem a organizações privadas e as outras duas a hospitais públicos e de ensino. Tais critérios foram estabelecidos para proporcionar uma comparação entre modelos de gestão distintos e também por conveniência dos pesquisadores.

A amostra foi constituída por enfermeiros e técnicos de enfermagem destas unidades e que obedeceram aos seguintes critérios de inclusão: livre aceitação para participar do estudo; estar presente no momento da coleta de dados sem previsão de férias ou licenças e possuir no mínimo seis meses de atuação profissional nestas UTIs.

A coleta de dados ocorreu no período de janeiro a abril de 2013. Incialmente, os enfermeiros foram abordados sobre a finalidade do estudo. Em seguida sortearam um técnico em enfermagem de sua equipe que foi consultado. Após a aceitação, todos assinaram o Termo de Consentimento Livre e Esclarecido. Destaca-se que o estudo foi apreciado pelo Comitê de Ética em Pesquisa da Universidade Federal de São Paulo com aprovação sob número 0839/10.

O enfermeiro não soube qual colaborador de sua equipe ele sorteou, já que os técnicos em enfermagem foram identificados com números que eram conhecidos apenas pelo pesquisador principal. Estes, no entanto, sabiam qual líder deveriam avaliar, pois o nome do enfermeiro estava escrito no instrumento de coleta. Garantiu-se o anonimato para impedir qualquer influência que pudesse interferir nas respostas.

Deixou-se com os enfermeiros num envelope dois instrumentos de coleta de dados: 1caracterização - com informações sobre idade, sexo, tempo de formação, trabalho na instituição e UTI, presença de pós-graduação e contato com o tema liderança; 2- Questionário Grid \& Liderança em Enfermagem: comportamento ideal ${ }^{(13)}$. Os técnicos em enfermagem sorteados também receberam um envelope contendo: 1- o mesmo instrumento de caracterização descrito acima; 2- Questionário Grid \& Liderança em Enfermagem: comportamento real ${ }^{(13)}$ considerando o enfermeiro que o sorteou. Todos os componentes da amostra foram orientados a preencher os instrumentos fora do ambiente de trabalho e a devolvê-los numa data previamente combinada.

Os dois questionários fundamentados nas Teorias Grid que visam avaliar o comportamento ideal de liderança do enfermeiro e o real na visão dos integrantes da equipe foram construídos e validados ${ }^{(13)}$. O primeiro é preenchido pelo líder e o segundo por um liderado. Uma vez que estes se adequam à realidade brasileira e passaram por validação aparente e de conteúdo, foram utilizados neste estudo.

Trata-se de 25 proposições com quatro possibilidades de respostas graduadas em pontos e assim discriminadas: totalmente desejável (quatro pontos), desejável (três pontos), indesejável (dois pontos) e totalmente indesejável (um ponto) ${ }^{(13)}$.

Cada afirmativa do instrumento se refere a um estilo de liderança. O estilo de maior pontuação relaciona-se à maneira como o enfermeiro exerce a sua liderança na sua concepção de comportamento ideal e na visão de um colaborador de sua equipe sobre o que é real ${ }^{(13)}$.

A taxa de retorno foi de 54,5\%, ou seja, houve 121 enfermeiros e 121 técnicos em enfermagem abordados. Porém 66 duplas constituíram a amostra sendo assim distribuídas: UTI A =34, UTI B=3, UTI $\mathrm{C}=16$ e UTI $\mathrm{D}=13$. Destaca-se que as UTI A e B são privadas e as C e D são públicas.

Os dados foram analisados com estatística descritiva e o teste de Wilcoxon para amostras independentes foi aplicado para verificar se existe diferença entre as percepções ideais e reais dos estilos de liderança dos enfermeiros das UTI dos hospitais públicos e privados. 
Dos 66 enfermeiros entrevistados, 48 (72,7\%) eram mulheres e trabalhavam nos seguintes turnos: 11 $(22,7 \%)$ manhã, quatro $(7,6 \%)$ manhã e tarde, oito (16,7\%) tarde e $25(53 \%)$ noite. O horário classificado como manhã e tarde deveu-se ao fato de uma das instituições possuir a carga horária de 12x36h durante o dia.

O contato com a liderança para estes enfermeiros foi de $100 \%$ na graduação, e também destacaram: palestras 29 (43,9\%) e treinamentos 28 (42,4\%). Além disso, 60 (90,9\%) possuíam alguma especialização, sendo a maioria em UTI 28 (46,6\%), e desses, 10 (35,7\%), possuíam outra especialização.

Já os técnicos em enfermagem entrevistados eram 41 (62,1\%) do sexo feminino. Trabalhavam em turnos iguais ou concomitantes com seus superiores e tiveram menos contato com o tema liderança: no curso técnico $42(63,6 \%)$, em palestras 18 (27,3\%), treinamentos 16 (24,3\%) e outros sete $(10,6 \%)$, além da graduação para aqueles que cursavam Enfermagem 11 (16,7\%).

As variáveis: idade, tempo de formação, trabalho na instituição e UTI estão descritas na Tabela 1, comparando os cargos. Os técnicos e enfermeiros trabalhavam na instituição e na UTI em média há cinco anos.

Dentre os 66 enfermeiros observados, 65 (98,5\%) consideraram que o estilo 9,9 de liderança era o ideal. Já entre as avaliações dos técnicos observou-se que nem todos os enfermeiros se portavam da forma como consideraram ideal conforme demostra a Tabela 2. Porém, 42 (63,6\%) foram avaliados como 9,9, com intervalo de confiança de $95 \%$ variando de $[50,8 \% ; 74,8 \%]$.

A Tabela 3 apresenta os estilos de liderança ideal e real dos enfermeiros considerando os hospitais privados (A e B) e públicos (C e D).

Tabela 1 - Descritivas das variáveis contínuas por cargo. São Paulo - SP, Brasil, 2013

\begin{tabular}{llcccc} 
Variável & Cargo & Mínimo & Máximo & Média & Desvio padrão \\
\hline \multirow{2}{*}{ Idade } & Enfermeiro & 23 & 53 & 32,9 & 7,1 \\
\cline { 2 - 6 } & Técnico & 21 & 59 & 34,7 & 8 \\
\hline \multirow{2}{*}{ Tempo de Formado } & Enfermeiro & 0,9 & 26 & 7,9 & 6,1 \\
\cline { 2 - 6 } & Técnico & 1 & 25 & 10 & 6,2 \\
\hline \multirow{2}{*}{ Tempo de Trabalho na Instituição } & Enfermeiro & 0,5 & 19 & 6 & 4,2 \\
\cline { 2 - 6 } & Técnico & 0,11 & 19 & 5,1 & 5 \\
\hline \multirow{2}{*}{ Tempo de Trabalho na UTI } & Enfermeiro & 0,3 & 19 & 5,2 & 4,1 \\
\cline { 2 - 6 } & Técnico & 0,11 & 19 & 4,8 & 4,9
\end{tabular}

Tabela 2 - Estilos de liderança real avaliados pelos técnicos em enfermagem. São Paulo, SP, Brasil, 2013

\begin{tabular}{lcc} 
Estilos de liderança real & $\mathbf{n}$ & $\mathbf{\%}$ \\
\hline 1,1 & 1 & 1,5 \\
\hline 1,9 & 6 & 9,1 \\
\hline 5,5 & 9 & 13,6 \\
\hline 9,1 & 8 & 12,1 \\
\hline 9,9 & 42 & 63,6 \\
\hline Total & 66 & 100
\end{tabular}

Tabela 3 - Estilos de liderança ideal e real dos enfermeiros considerando os hospitais privados e públicos. São Paulo, SP, Brasil, 2013

\begin{tabular}{|c|c|c|}
\hline Hospitais & $\begin{array}{c}\text { Estilo de } \\
\text { Liderança Ideal }\end{array}$ & $\begin{array}{c}\text { Estilo de } \\
\text { Liderança Real }\end{array}$ \\
\hline \multirow{5}{*}{$\begin{array}{l}\text { Privados }(\mathrm{n}=36 \\
\text { duplas) }\end{array}$} & $1,1=0$ & $1,1=1(2,8 \%)$ \\
\hline & $1,9=0$ & $1,9=2(5,5 \%)$ \\
\hline & $5,5=0$ & $5,5=5(13,9 \%)$ \\
\hline & $9,1=0$ & $9,1=5(13,9 \%)$ \\
\hline & $9,9=36(100 \%)$ & $9,9=23(63,9 \%)$ \\
\hline \multirow{5}{*}{$\begin{array}{l}\text { Públicos ( } \mathrm{n}=30 \\
\text { duplas) }\end{array}$} & $1,1=0$ & $1,1=0$ \\
\hline & $1,9=0$ & $1,9=4(13,3 \%)$ \\
\hline & $5,5=1(3,3 \%)$ & $5,5=4(13,3 \%)$ \\
\hline & $9,1=0$ & $9,1=3(10 \%)$ \\
\hline & $9,9=29(96,7 \%)$ & $9,9=19(63,3 \%)$ \\
\hline
\end{tabular}


O teste de Wilcoxon para amostras independentes foi aplicado para verificar se havia diferença nas percepções ideais e reais dos estilos de liderança dos enfermeiros das UTI dos hospitais privados e públicos. Obteve-se $p=0,67$ o que não é significante estatisticamente.

\section{DISCUSSÃO}

Do ponto de vista da caracterização da amostra, houve predominância do sexo feminino tanto para os enfermeiros quanto para os técnicos em enfermagem. A enfermagem é uma profissão predominantemente exercida pelas mulheres. Muito embora exista uma tendência de inversão, as amostras de outros estudos ${ }^{(13-15)}$ que utilizaram método similar ao adotado nesta pesquisa também encontraram esta realidade.

O contato com o tema liderança obteve maior destaque nos cursos de graduação e técnico. As outras modalidades tais como palestras e treinamentos evidenciaram-se principalmente entre os enfermeiros. Espera-se que o maior enfoque na capacitação da competência liderança ocorra com estes profissionais uma vez que são os responsáveis pela condução dos times assistenciais na busca dos melhores resultados. Por isto as organizações devem criar uma estrutura que promova o desenvolvimento do líder continuamente.

Observou-se também que os enfermeiros possuíam curso de especialização com especial destaque para UTI. A necessidade de aprimorar o conhecimento para fundamentar a prática assistencial torna-se uma necessidade atual. Isto é corroborado por estes achados. O próprio ambiente de trabalho instiga os enfermeiros a buscarem uma capacitação para tornar a assistência fundamentada e que atenda às necessidades do paciente e equipe.

Os técnicos em enfermagem possuíam maior idade que os enfermeiros bem como tempo de formação. No entanto, trabalham há menos tempo na instituição e UTI. Liderar este grupo é um desafio. Sendo jovem e com menos experiência, o enfermeiro necessita mostrar sua liderança. Estabelecer um elo de confiança e colocar o seu conhecimento a serviço da equipe são pressupostos que necessitam ser exercidos com maestria.

A maioria dos enfermeiros considerou o estilo 9,9 como sendo o ideal para o exercício da liderança. O líder desse estilo faz um esforço vigoroso e consegue adesão entusiasta. Solicita e dá atenção a ideias, opiniões e atitudes diferentes, reavalia continuamente os próprios dados, assegurando sua validade. Acha importante expressar suas preocupações, muda o modo de pensar frente às melhores ideias, procura saber os motivos dos conflitos para solucioná-los, valoriza decisões certas, o entendimento e o acordo, encorajando o feedback de mão dupla ${ }^{(13,15)}$.

As palavras chave que destacam esse comportamento são: sincero e direto, confiante, decidido, determinado, gosta de trabalhar, busca os fatos, focaliza problemas reais, vai até o fim, ventila problemas, possui espírito realizador, altos padrões, identifica causas obscuras, inovador, tem mente aberta. As prioridades são claras, estabelece metas desafiadoras, externa ideias, espontâneo, estimula a participação e também é altruísta ${ }^{(13,15)}$.

Evidentemente que neste estilo de liderança o líder está preocupado com os interesses hospitalares e também com os da equipe ${ }^{(13)}$. As organizações procuram líderes com esta performance e, o enfermeiro pesquisado identifica-se com esta proposta de trabalho. Outros estudos ${ }^{(13-15)}$ também encontraram este resultado.

Considerando que o enfermeiro deseja ser este líder 9,9, como ele está preparando-se para isto? As organizações de saúde estão oferecendo subsídios para proporcionar o alcance deste objetivo? Estas questões são importantes para serem respondidas e principalmente devem subsidiar novos estudos nesta área, pois identificar o estilo ideal é necessário, porém o meio de atingi-lo é o grande desafio do líder e de suas instâncias superiores.

O preparo para liderança foi abordado num estudo bibliométrico ${ }^{(16)}$ que procurou identificar como se encontra a produção científica nesta área. Os resultados mostraram que há muitas estratégias exitosas, porém ainda não se verifica qual o seu impacto na formação do líder. Há necessidade de 
instigar a busca de evidências científicas neste campo do conhecimento para possibilitar aos gestores programas de treinamento e desenvolvimento que possibilitem o desenvolvimento desta competência.

Quando se observou a liderança real, a maioria dos enfermeiros foi avaliada como 9,9 pelo técnico em enfermagem. Isto demonstra correspondência de opinião muito interessante. $\mathrm{O}$ enfermeiro foi identificado como um líder que procura trabalhar em equipe, respeitar os seus colaboradores e principalmente preocupar-se com o seu desenvolvimento ${ }^{(13)}$. Isto demonstra o quanto esta equipe encontra-se homogênea.

Corroborando este achado, a liderança eficaz do enfermeiro melhora a qualidade da assistência de enfermagem na medida em que inspira e motiva a equipe a aceitar ações inovadoras e transformadoras. Além disto, incentiva o compromisso e a mudança, aumenta a relação de confiança entre líder e os liderados e garante a eficácia no atendimento por meio da satisfação no trabalho ${ }^{(17)}$.

Entretanto, também se verificou a distribuição de outros estilos de liderança reais 5,5 e 9,1. Estes enfermeiros, avaliados pelos seus liderados, estão mais preocupados com os interesses hospitalares em detrimento aos da equipe ${ }^{(13)}$. Em distribuição menor, encontrou-se também, 1,9 e 1,1, cuja preocupação centra-se mais com os anseios da equipe do que com os objetivos da organização ${ }^{(13)}$.

É notório perceber que prevalecem os interesses hospitalares ${ }^{(13)}$. O fato da amostra de enfermeiros ser jovem pode contribuir para este achado. Uma vez que a liderança está em processo de construção, é mais fácil cumprir as normas hospitalares do que atender aos anseios da equipe. Além disto, sabese que a imaturidade profissional pode ser considerada um obstáculo à liderança, já que o seu aprimoramento se dá ao longo do tempo e com as experiências vivenciadas ${ }^{(18)}$. Portanto, a busca para o seu desenvolvimento, por parte dos enfermeiros e também da instituição, é fundamental.

Outro achado deste estudo é que não houve diferença entre os estilos de liderança ideal e real dos enfermeiros da UTI de hospitais privados e públicos mesmo considerando os modelos de gestão diferentes adotados nestas instituições.

O contexto do cuidado intensivo tem algumas características peculiares. Estes ambientes costumam proporcionar um trabalho integrado e dinâmico entre os membros do time. A gravidade do paciente e a assertividade nas tomadas de decisão favorecem uma liderança participativa. Isto descentraliza o poder e a autoridade, permitindo a elaboração de estratégias compartilhadas de resolução de problemas $^{(10)}$. Estas variáveis poderiam explicar este resultado ${ }^{(3)}$. Porém novos estudos precisam ser desenvolvidos comparando estes setores com outros do ambiente hospitalar para fundamentar esta afirmação.

\section{- CONCLUSÃO}

Este estudo possibilitou mapear quais são os estilos de liderança dos enfermeiros intensivistas e verificar que pertencer a hospitais privados e públicos não interferiu no exercício desta competência.

As limitações desta pesquisa centram-se no fato de que a Teoria do Grid Gerencial é da década de 1990 e não foi aplicada no contexto da unidade de terapia intensiva. Além disto, também se destaca o tamanho da amostra. Porém, outras possibilidades de estudo podem surgir a partir desta investigação: descobrir se há relações entre a liderança e os modelos assistenciais e gerenciais em estudos multicêntricos e com amostras maiores; desenvolvimento de programas de treinamento para o enfermeiro líder no contexto do cuidado intensivo, dentre outros. Assim fundamentar-se-á cada vez mais esta área do conhecimento e poderemos oferecer aos enfermeiros oportunidades de aprimorar a liderança tão necessária e requerida atualmente.

\section{- APOIO FINANCEIRO}

Coordenação de Aperfeiçoamento de Pessoal de Nível Superior (CAPES), Brasil, processo $\mathrm{n}^{\circ}$ 008898340001-08. 


\section{REFERÊNCIAS}

1. Balsanelli AP, Cunha ICKO, Whitaker IY. Estilos de liderança de enfermeiros em unidade de terapia intensiva: associação com perfil pessoal, profissional e carga de trabalho. Rev Latino-Am Enfermagem. 2009; 17(1):28-33.

2. Balsanelli AP, Cunha ICKO, Whitaker IY. Estilos de liderança e perfil professional de enfermeiros em Unidade de Terapia Intensiva. Acta Paul Enferm. 2008; 21(2):300-4.

3. Balsanelli AP, Cunha ICKO. Liderança do enfermeiro em unidade de terapia intensiva e sua relação com ambiente de trabalho. Rev. Latino-Am. Enfermagem. 2015; 23(1):106-13.

4. Rosengren K, Bondas T, Nordholm L, Nordstrom G. Nurses' views of shared leadership in ICU: a case study. Intensive Crit Care Nurs. 2010; 26(4):226-33.

5. Rosengren K, Athlin E, Segesten K. Presence and availability: staff conceptions of nursing leadership on na intensive care unit. J Nurs Manag. 2007; 15(5):522-29.

6. Linton J, Farrell MJ. Nurses' perceptions of leadership in an adult intensive care unit: a phenomenology study. Intensive Crit Care Nurs. 2009; 25(2):64-71.

7. Gelbcke FL, Souza LA, Sasso GMD, Nascimento E, Bulb MBC. Liderança em ambientes de cuidados críticos: reflexões e desafios à enfermagem brasileira. Rev. bras. enferm. 2009; 62(1):136-9.

8. Camelo SHH. Professional competences of nurse to work in Intensive Care Units: an integrative review. Rev. Latino-Am. Enfermagem 2012; 20(1):192-20.

9. Bernardes A, Cummings G, Évora YDM, Gabriel CS. Participatory management model in a public hospital. Rev. Latino-Am. Enfermagem 2012; 20(6):1142-51.

10. Bernardes A, Cecilio LCO, Évora YDM, Gabriel CS, Carvalho MB. Modelo de gestão colegiada e descentralizada em hospital público: a ótica da equipe de enfermagem. Rev. Latino-Am. Enfermagem. 2011; 19(4):1003-10.

11. Amestoy SC, Backes VMS, Trindade LL, Canever BP. Produção científica sobre liderança no contexto da enfermagem. Rev. esc. enferm. USP. 2012; 46(1):227-33.

12. Blake R, Mouton J, Tapper M. Grid approaches for managerial leadership in nursing. In: Leddy S, Pepper JM. Conceptual bases of professional nursing. Philadelphia (USA): Lippincott Co; 1985. p.2-20.

13. Higa EFR, Trevisan MA. Os estilos de liderança idealizados pelos enfermeiros.Rev. Latino-am Enfermagem 2005; 13(1):59-64.

14. Santos I, Castro CB. Estilos e dimensões da liderança: iniciativa e investigação no cotidiano do trabalho de enfermagem hospitalar. Texto Contexto Enferm. 2008; 17(4):734-42.

15. Trevizan MA, Mendes IAC, Hayashida M, Galvão CM, Cury SRR. Análise de expectativas sobre a liderança do enfermeiro à luz das teorias Grid. Rev. gaúcha Enferm. 2001; 22(1):20-9.

16. Neves VR, Sanna MC. Ensino da liderança em enfermagem: um estudo bibliométrico. Acta Paul Enferm. 2012; 25(2):308-13.

17. Botma Y, Botha H, Nel M. Transformation: are nurse leaders in critical care ready? J. Nurs. Manag. 2011; 20:92127.

18. Paula GF, Figueiredo ML, Camargo FC, Iwamoto HH, Caixeta CRCB. Concepções de liderança entre enfermeiros assistenciais de um hospital do norte de Minas Gerais. Rev. eletrônica. enferm. [Internet] 2012; 14(4) [acesso em 03 dez 2015]. Disponível: http://revistas.jatai.ufg.br/index.php/fen/article/view/15102 